\title{
Device-measured physical activity and sedentary behaviour in relation to mental wellbeing: An analysis of the 1970 British Cohort Study.
}

\author{
Nipuna Senaratne ${ }^{1}$, Brendon Stubbs ${ }^{2,3}$, André O Werneck ${ }^{4}$, Emmanuel Stamatakis $^{5}$, Mark Hamer $^{1}$ \\ ${ }^{1}$ Institute Sport Exercise \& Health, Division of Surgery \& Interventional Science, Faculty of Medical \\ Sciences, University College London, London, UK \\ ${ }^{2}$ Department of Psychological Medicine, Institute of Psychiatry, Psychology and Neuroscience, King's \\ College London, De Crespigny Park, London, Box SE5 8AF, United Kingdom. \\ ${ }^{3}$ South London Maudsley NHS Foundation Trust, London, UK. \\ ${ }^{4}$ Department of Nutrition, School of Public Health, University of São Paulo (USP), São Paulo; Brazil. \\ ${ }^{5}$ Charles Perkins Centre, School of Health Sciences, Faculty of Medicine and Health, University of \\ Sydney, Sydney, Australia.
}

Corresponding author: Nipuna Senaratne, Institute Sport Exercise \& Health, 170 Tottenham Court Road, University College London, London WC1E 6BT. (E-mail: nipuna.senaratne.19@ucl.ac.uk)

This work was funded by British Heart Foundation grant SP/15/6/31397, and a joint award from Economic Social Research Council and Medical Research Council, grant RES-579-47-0001.

Conflict of interest: ES and MH received an unrestricted grant from PAL Technologies, Scotland, UK.

\section{Word Counts}

Abstract: 243

Main Text: 3456

\section{Keywords:}

physical activity; moderate-to-vigorous physical activity; sitting time; mental wellbeing; mental health 


\section{Abstract}

Although physical activity and sedentary behaviour have established associations with mental illness, the extent to which they impact on mental wellbeing is not well understood. We examined associations between moderate to vigorous physical activity (MVPA), sitting time (ST) and mental wellbeing in 4,526 participants from the 1970 British Cohort Study (UK) in the age 46 survey (201618). MVPA and ST were measured using a thigh mounted accelerometer device (activPAL 3 micro) worn continuously for 7 days and participants completed the 14-item Warwick-Edinburg Mental Wellbeing Scale (WEMWBS) to assess mental wellbeing. In linear regression models MVPA (per hr) was associated with an additional 0.57 points on the WEMWBS (95\% $\mathrm{Cl} 0.03-1.12)$ regardless of gender, wear time, education, socioeconomic status, smoking, body mass index, disability and psychological distress. ST was not associated with WEMWBS in the adjusted models $(B=-0.11,-0.23$, 0.02). In MVPA stratified analyses, ST showed a linear trend with WEMBS in participants with low levels of MVPA but not in medium and high MVPA categories. In this large, nationally representative cohort, device-measured MVPA showed an association with higher mental wellbeing whilst ST was only associated with reduced mental wellbeing in participants with low levels of MVPA. Our main limitation was the cross-sectional design which precludes any inference of direction of association or causality. Nevertheless, interventions to promote MVPA may be an effective public health policy to promote mental wellbeing. Further investigation of the effect different sitting behaviours has on mental wellbeing is warranted. 


\section{Introduction}

Mental wellbeing is a state of positive psychological health that allows individuals and communities to function well ${ }^{1}$. It can be conceptualised through two approaches: a hedonic perspective relating to subjective experiences of happiness and perceived life satisfaction and a eudaimonic perspective focusing on maximising human potential and functioning ${ }^{2}$. The World Health Organisation (WHO) recognises that positive mental wellbeing 'work productively and fruitfully and make a contribution to their community ${ }^{3}$ whilst wellbeing is also associated with reduced mortality in healthy and chronic disease populations ${ }^{4}$.

Physical activity and sedentary behaviour (defined as a sitting or reclining posture with low energy expenditure) are important determinants of physical and psychological health. Physical activity is linked to prevention and treatment of mental illness ${ }^{5,6}$ and moderate-to-vigorous physical activity (MVPA) is most beneficial ${ }^{7}$. However, the link between MVPA and mental wellbeing is not well established: device-measured MVPA has been associated with mental wellbeing in over $65 \mathrm{~s}^{8}$ but not in adults ${ }^{9}$ or adolescents ${ }^{10}$. Sedentary behaviour is associated with mental illness ${ }^{11}$ but evidence for an association with mental wellbeing is also mixed: self-reported screen time correlates with wellbeing $^{12,13,14}$ but device-measured sitting time (ST) has not shown an association, ${ }^{15}$.

Current evidence is limited by small sample sizes and reliance on self-reported behaviour, which has known biases ${ }^{16}$. Wearable accelerometer devices have been used but have limitations. Some use a count-based threshold to classify sedentary behaviour ${ }^{17}$ which can misclassify low intensity nonsedentary behaviours such as standing and sleep ${ }^{18,19,20}$, whilst there are concerns over the accuracy of sitting estimates from wrist and hip worn monitors, ${ }^{20}$. These limitations can be addressed using methods that directly estimate body posture, such as thigh mounted accelerometry ${ }^{19}$.

This study investigated whether device-measured MVPA and ST are associated with mental wellbeing and aimed to overcome preceding limitations by using data from a large national cohort study where MVPA and ST were assessed using thigh-mounted accelerometry. 


\section{Methods}

\section{i) Participants}

The 1970 British Cohort Study consists of 17,196 people who were born in the UK during a single week in 1970 and followed up regularly throughout childhood and adulthood ${ }^{21,22}$. This analysis utilises data from the age 46 survey (2016-2018): which involved interviews and biomedical assessments ${ }^{23}$. Participants provided informed consent and ethical approval was obtained from NRES Committee South East Coast - Brighton \& Sussex (Ref 15/LO/1446).

\section{ii) Measures of Physical Activity and Sedentary Behaviour}

MVPA and ST were measured using a thigh-mounted accelerometer device (activPAL3 micro; PAL Technologies Ltd., Glasgow, $\mathrm{UK}^{24}$ ) using a previously described wear protocol ${ }^{25}$. The device was fitted on the upper thigh and participants were advised to wear it continuously for 7 days including during sleep, bathing, and all physical activity. The activPAL uses thigh inclination and acceleration to estimate body posture (calculating 1-hour periods as sitting/lying, standing and walking), transition between postures, stepping and stepping speed (cadence). From this ST (hr/d) was calculated. The thigh mounted activPAL has been validated in measuring ST compared to direct observation ${ }^{26}$ and has been shown to be more precise and sensitive to changes in sitting time than hip worn monitors ${ }^{15}$. MVPA ( $\mathrm{hr} / \mathrm{d}$ ) was derived using a step cadence threshold of $\geq 100^{27}$. Valid waking wear time was calculated using a previously validated algorithm which distinguishes sleep and prolonged non-wear ${ }^{28}$. Data on common types of MVPA and ST was obtained using self-reported questionnaires.

\section{iii) Measures of Mental Wellbeing}

Wellbeing was assessed using the Warwick-Edinburgh Mental Wellbeing Scale (WEMWBS), a 14-item scale focussing on positive hedonic and eudaimonic aspects of mental health (Supplementary Table $1)^{29}$. The scale provides a score from 14-70, with higher scores reflecting greater wellbeing. The WEMWBS has good content and criterion validity, internal consistency and test-retest reliability ${ }^{25}$ and has been validated in adults, adolescents, ethnic minorities and secondary mental health service users across the UK and Europe ${ }^{30}$.

\section{iii) Covariates}

Potential confounders were chosen from known correlates of MVPA/ST that may influence mental wellbeing including gender, health status and behaviours, education and socioeconomic status ${ }^{31}$. The covariates used included: occupation (National Statistics Socio-economic classification (NS-SEC): 
professional/managerial; technical; manual; unemployed; not classifiable not stated), highest educational qualification obtained (non-degree educated; degree educated), smoking status (never smoked; ex-smoker; current smoker), disability (European Statistics of Income and Living Condition (EU-SILC) classification: none; some extent; severely hampered ${ }^{32}$, body mass index $\left(\mathrm{BMI} \mathrm{kg} / \mathrm{m}^{2}\right.$ ) and Malaise Inventory Score (a 9-item questionnaire assessing symptoms of psychological stress/depression/anxiety, Supplementary Table 2). The Malaise Inventory assesses important covariates that are distinct to mental wellbeing ${ }^{33,34}$ and has shown robust psychometric properties ${ }^{35}$.

iv) Statistical Analyses

Associations between MVPA, ST (continuous data) and WEMWBS were examined using general linear regression models. We adjusted initially for gender and waking wear time (Model 1), additionally for highest educational qualification obtained, occupation, smoking, BMI and disability (Model 2) and additionally for Malaise Inventory (Model 3). Associations between self-reported activities and wellbeing were examined using linear models using similar covariates to Model 3 . In order to examine joint associations, MVPA and ST were each categorised into three equal tertiles: MVPA $(<0.6 \mathrm{hr} / \mathrm{d}, 0.6$ $1 \mathrm{hr} / \mathrm{d},>1 \mathrm{hr} / \mathrm{d}$ ) and ST ( $<8 \mathrm{hr} / \mathrm{d}, 8-10 \mathrm{hr} / \mathrm{d},>10 \mathrm{hr} / \mathrm{d})$. These were combined to produce nine groups (i.e. low MVPA / high ST; low MVPA / medium ST etc...). Linear models were used to examine differences in WEMBS between groups adjusting initially for gender and subsequently for all covariates. 


\section{Results}

After excluding missing covariate and outcome data the final analysis comprised 4,526 participants: 2163 men and 2364 women (Figure 1). Participants declining to wear the device (11.8\%) were more likely to be male, smokers, report poorer health, and be obese ${ }^{27}$. Descriptive characteristics for the included sample are shown in Table 1. The mean WEMWBS score overall was $50.26 \pm 8.35$ and did not differ with gender. Mean MVPA and ST were $50.99 \pm 25.42 \mathrm{mins} / \mathrm{d}$ and $9.25 \pm 1.96 \mathrm{hr} / \mathrm{d}$ respectively. Gender, smoking, disability and malaise score were associated with WEMWBS scores (Supplementary Table 3).

In gender and wear time adjusted analyses (Model 1) MVPA showed a significant association with mental wellbeing (Table 2), with an extra hour per day of MVPA adding 2.15 additional points on the WEMWBS $(95 \% \mathrm{Cl} 1.53-2.78, \mathrm{p}<0.01)$. After further adjusting for educational status, social occupational position, smoking, BMI and disability (Model 2) MVPA remained associated with higher WEMWBS scores with an extra hour per day adding 0.92 points ( $95 \% \mathrm{Cl} 0.29-1.54, \mathrm{p}<0.01)$. In Model 3 , where malaise score was also adjusted for, MVPA remained associated with higher mental wellbeing: an extra hour per day providing 0.57 additional points on the WEMWBS ( $95 \% \mathrm{Cl}$ 0.03-1.12, $p=0.04$ ). ST was not associated with lower mental wellbeing in any of the models (Table 2 ). In analyses utilising context specific data, wellbeing was negatively associated with self-reported TV viewing and positively with walking for pleasure (Supplementary Table 4).

When analysing joint associations between sitting and MVPA, we observed a linear trend for sitting and WEMBS only in the lowest tertile of MVPA $(p<0.001)$ when adjusted for covariates. We observed no consistent pattern in the medium and high MVPA categories (Figure 2). 


\section{Discussion}

Device-measured MVPA was associated with higher mental wellbeing and this association was not nullified following adjustments for gender, wear time, educational status, social occupational position, smoking, BMI, disability and psychological distress. To our knowledge this is the first large scale cohort study showing an association between device-measured MVPA and mental wellbeing. A 1-unit increase in WEMBS is clinically relevant, correlating with reductions in suicidal thoughts and depressive or anxiety symptoms ${ }^{36}$. Our demonstrated benefits of an additional hour of MVPA per day therefore may have relevance for population health. However, controlled trials are needed to establish causality and a meaningful effect size, which would fully elucidate any public health implications.

Physical activity and sitting are not mutually exclusive; time in one activity likely displaces another. Greater ST was associated with lower wellbeing only in participants recording lower MVPA levels. At higher levels, the association of sitting with wellbeing was markedly attenuated. Participants with highest levels of MVPA displayed the greatest wellbeing scores regardless of ST. Our findings may explain why pre-existing evidence linking ST with mental wellbeing has been mixed ${ }^{12,13,14,15,16}$ and mirror physical health outcomes including all-cause mortality and cardiovascular events where associations with ST are dependent on MVPA ${ }^{37}$. The lack of independent association between ST and wellbeing may reflect differing types of sedentary activities. Consistent with our findings, mentallypassive activities (e.g. TV-viewing) are associated with lower wellbeing, whilst mentally-active activities (e.g. reading a book) are not, and can even be protective ${ }^{38,39,13,14}$. Interventions focussing on reducing ST alone may not be effective in enhancing mental wellbeing without targeting negative sitting behaviours and promoting reallocation of that time to physically or mentally-active behaviours.

The association of MVPA and ST with wellbeing likely arises from cognitive, biological and social mechanisms ${ }^{40}$. MVPA can be associated with higher cognition and reduction in stress levels, which are interrelated with mental wellbeing ${ }^{41}$. Among biological mechanisms, both MVPA and lower ST are associated with reduced inflammation ${ }^{42}{ }^{43}$, and MVPA with cortisol release, both of which are associated with higher wellbeing ${ }^{44}, 45$. Neuroplasticity mediated by brain-derived neurotrophic factor (BDNF) and structural changes in hippocampal volume may also drive part of the association between MVPA, ST and higher wellbeing ${ }^{46,47,48}$. In addition, MVPA as part of group activities is associated with higher social connectedness and social support, increasing mental wellbeing ${ }^{49,50}$.

Our study has several significant limitations. Data are cross-sectional which does not allow for any causality inference. People with poor self-rated wellbeing may be more sedentary therefore the 
direction of association is unclear. Interventional studies are therefore needed. As with all cohorts there were losses during follow-up and health-related and socioeconomic factors that were used as covariates are associated with dropout. Social support was not assessed but is known to be associated with both physical activity ${ }^{51}$ and mental wellbeing ${ }^{52}$. Objectively measured physical activity may also imprecisely capture certain activities such as swimming and cycling, although the prevalence of these were low in our cohort. Finally, the lack of age variation of participants limits generalisability to other demographic groups such as children, adolescents or the elderly.

Nevertheless, our study has a number of strengths. This is a large-scale analysis from a nationally representative birth cohort. Physical activity and sitting were device-measured using thigh-based accelerometers and validated protocols were used to calculate ST and MVPA. Finally, we used a wellestablished measure of mental wellbeing and obtained mean scores consistent with UK population norms $s^{53}$.

Our findings may have important policy implications. Promoting MVPA to WHO recommended levels ${ }^{54}$ may be an effective public health strategy to improve mental-wellbeing and should be a key focus of mental health policy. Reducing ST may not be as effective in promoting mental wellbeing but should be encouraged, especially in populations with low levels of MVPA. However, it is likely that not all sitting is the same and investigation of the difference between mentally-active and passive sitting is needed in order to establish targets for policy intervention. 


\section{Acknowledgements}

Funding sources

This work was funded by British Heart Foundation grant SP/15/6/31397, and a joint award from Economic Social Research Council and Medical Research Council, grant RES-579-47-0001. The funders had no role in the study design; in the collection, analysis and interpretation of data; in writing of the report; or in the decision to submit the paper for publication.

Author contributions

MH had full access to the data and takes responsibility for the integrity and accuracy of the results. NS and MH performed the analyses and drafted the paper. All authors contributed to the concept and design of the study and critical revision of the manuscript. 
Table 1: Demographic and covariate information for study participants

\begin{tabular}{|c|c|c|c|c|c|c|c|}
\hline \multirow{3}{*}{ WEMWBS Score } & & \multicolumn{2}{|c|}{ Total Sample $(n=4527)$} & \multicolumn{2}{|c|}{ Male $(n=2163)$} & \multicolumn{2}{|c|}{ Female $(n=2364)$} \\
\hline & & Mean / n & SD / \% & Mean / n & SD / \% & Mean / n & SD / \% \\
\hline & & 50.56 & 8.35 & 50.22 & 8.17 & 50.87 & 8.50 \\
\hline $\operatorname{MVPA}(\mathrm{min} / \mathrm{d})$ & & 50.99 & 25.42 & 50.34 & 26.26 & 51.59 & 24.61 \\
\hline $\mathrm{ST}(\mathrm{hr} / \mathrm{d})$ & & 9.25 & 1.96 & 9.51 & 1.96 & 9.02 & 1.93 \\
\hline \multicolumn{8}{|l|}{ COVARIATES } \\
\hline \multicolumn{2}{|c|}{ Device Wear Time (hr/d) } & 15.82 & 1.25 & 15.98 & 1.24 & 15.67 & 1.24 \\
\hline \multirow{2}{*}{ Education } & Non-degree educated & 3318 & 73.3 & 1571 & 34.7 & 673 & 14.9 \\
\hline & Degree educated & 1209 & 26.7 & 592 & 13.1 & 617 & 13.6 \\
\hline \multirow{4}{*}{$\begin{array}{l}\text { Social } \\
\text { Occupational } \\
\text { Position }\end{array}$} & Professional / Managerial & 1934 & 42.7 & 907 & 41.9 & 1027 & 43.3 \\
\hline & Technical & 1254 & 27.7 & 627 & 29.0 & 627 & 26.5 \\
\hline & Manual & 580 & 12.8 & 265 & 12.3 & 315 & 13.3 \\
\hline & Unemployed & 92 & 2.0 & 45 & 2.1 & 47 & 2.0 \\
\hline
\end{tabular}

Page 10 of 24 


\begin{tabular}{|c|c|c|c|c|c|c|c|}
\hline & Not classifiable or not stated & 667 & 14.7 & 319 & 14.7 & 348 & 14.7 \\
\hline \multirow{3}{*}{ Smoking } & Never smoked & 2279 & 50.3 & 1076 & 49.7 & 1203 & 50.9 \\
\hline & Ex-smoker & 1455 & 32.1 & 688 & 31.8 & 767 & 32.4 \\
\hline & Current smoker & 793 & 17.5 & 399 & 18.4 & 394 & 16.7 \\
\hline \multicolumn{2}{|l|}{$\mathrm{BMI}\left(\mathrm{kg} / \mathrm{m}^{2}\right)$} & 28.20 & 5.36 & 28.49 & 4.6 & 27.94 & 5.95 \\
\hline \multirow{3}{*}{ Disability } & No long-standing health condition & 3854 & 85.1 & 1893 & 87.5 & 1961 & 83.0 \\
\hline & To some extent & 455 & 10.1 & 178 & 8.2 & 277 & 11.7 \\
\hline & Severely hampered & 218 & 4.8 & 92 & 4.3 & 126 & 5.3 \\
\hline \multirow{2}{*}{$\begin{array}{l}\text { Malaise } \\
\text { Inventory }\end{array}$} & Low malaise (0-3) & 3759 & 83.0 & 1848 & 85.4 & 1911 & 80.08 \\
\hline & High malaise $(4+)$ & 768 & 17.0 & 315 & 14.6 & 453 & 19.2 \\
\hline
\end{tabular}

WEMWBS, Warwick-Edinburgh Mental Wellbeing Scale. MVPA, moderate to vigorous physical activity. ST, total daily sitting time (or sitting time). BMI, body mass index. Malaise Inventory - a 9-item questionnaire that assess for symptoms of psychological stress, anxiety and depression (see supplementary table 2). 
Table 2: Association of moderate to vigorous physical activity and sitting time with mental wellbeing

\begin{tabular}{|c|c|c|c|c|}
\hline & & \multicolumn{3}{|c|}{ Mean difference in WEMWBS Score } \\
\hline & & Model 1 & Model 2 & Model 3 \\
\hline \multirow{3}{*}{ MVPA } & B & 2.15 & 0.92 & 0.57 \\
\hline & $95 \% \mathrm{Cl}$ & $1.53-2.78$ & $0.29-1.54$ & $0.03-1.12$ \\
\hline & $p$ & $<0.01$ & $<0.01$ & 0.04 \\
\hline \multirow{3}{*}{ ST } & B & -0.14 & -0.08 & -0.11 \\
\hline & $95 \% \mathrm{Cl}$ & $-0.28-0.01$ & $-0.22-0.06$ & $-0.23-0.02$ \\
\hline & $p$ & 0.06 & 0.27 & 0.09 \\
\hline
\end{tabular}

WEMWBS, Warwick-Edinburgh Mental Wellbeing Scale. MVPA, moderate to vigorous physical activity (hr/d). ST, sitting time ( $\mathrm{hr} / \mathrm{d})$. Cl, confidence interval. Model 1: adjusted for wear time and gender. Model 2: adjusted for wear time, gender, education, social occupational position, smoking status, BMI, disability. Model 3: adjusted for wear time, gender, education, social occupational position, smoking status, BMI, disability and malaise score. 
Figure 1: Flowchart for inclusion and exclusion of participants

$$
\begin{aligned}
& \text { Participants approached to wear } \\
& \text { activPAL } \\
& n=7,439
\end{aligned}
$$
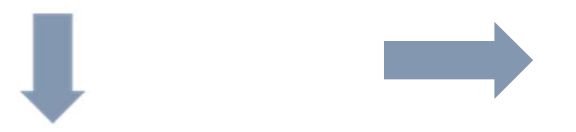

Declined to wear activPAL, $\mathrm{n}=877$

Participants consented to

wearing activPAL

$$
n=6,562
$$
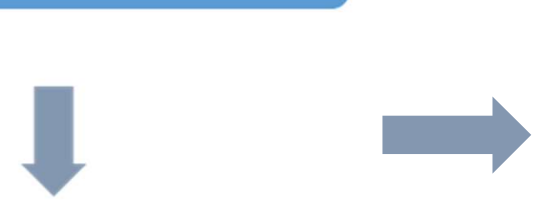

Unusable data, $n=1,670$

Nurse unable to initiate, $n=102$

Lost in post, $\mathrm{n}=591$

Unable to download, $n=272$

Data available for less than one valid day, $n=149$

Unable to compute physical activity based on cadence, $n=556$

Useable activPAL data

$n=4,892$
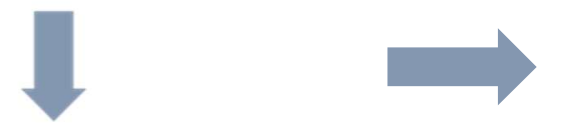

ncomplete co-variate / WEMWB

data, $n=366$

$$
\begin{aligned}
& \text { Useable activPAL, co-variate and } \\
& \text { WEMWB data } \\
& \qquad n=4,526
\end{aligned}
$$

WEMWBS, Warwick-Edinburgh Mental Well-being Scale. 
Figure 2: Adjusted combined association of physical activity and sitting time on wellbeing.

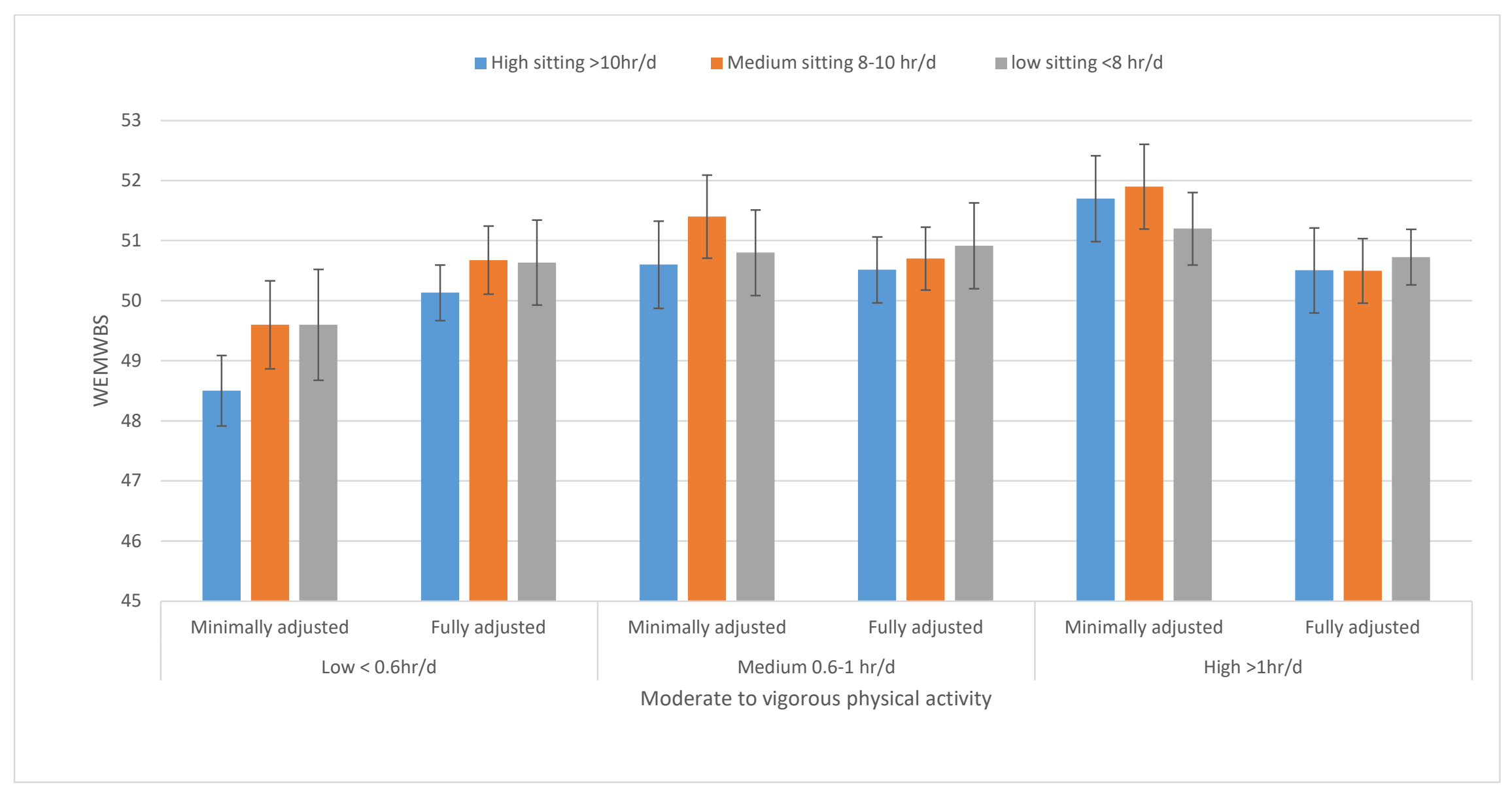

Data are presented as marginal mean WEMWBS scores with 95\% confidence intervals minimally adjusted (for gender only) or fully adjusted (for gender, education, social occupational position smoking, disability, BMI, malaise). MVPA and sitting categories reflect tertiles. WEMWBS, Warwick-Edinburgh Mental Wellbeing Scale. 
Supplementary Table 1: The Warwick Edinburgh Mental Wellbeing Scale

\begin{tabular}{|c|c|c|c|c|c|}
\hline Questions & None of the time & Rarely & Some of the time & Often & All of the time \\
\hline I've been feeling optimistic about the future & 1 & 2 & 3 & 4 & 5 \\
\hline I've been feeling useful & 1 & 2 & 3 & 4 & 5 \\
\hline I've been feeling relaxed & 1 & 2 & 3 & 4 & 5 \\
\hline I've been feeling interested in other people & 1 & 2 & 3 & 4 & 5 \\
\hline I've had energy to spare & 1 & 2 & 3 & 4 & 5 \\
\hline I've been dealing with problems well & 1 & 2 & 3 & 4 & 5 \\
\hline I've been thinking clearly & 1 & 2 & 3 & 4 & 5 \\
\hline I've been feeling good about myself & 1 & 2 & 3 & 4 & 5 \\
\hline I've been feeling close to other people & 1 & 2 & 3 & 4 & 5 \\
\hline I've been feeling confident & 1 & 2 & 3 & 4 & 5 \\
\hline I've been able to make up my own mind about things & 1 & 2 & 3 & 4 & 5 \\
\hline I've been feeling loved & 1 & 2 & 3 & 4 & 5 \\
\hline I've been interested in new things & 1 & 2 & 3 & 4 & 5 \\
\hline I've been feeling cheerful & 1 & 2 & 3 & 4 & 5 \\
\hline
\end{tabular}

Scores for the 14 individual questions are summed to provide an overall score from 14-70 with greater scores reflecting higher levels of wellbeing. 
Supplementary Table 2: The Malaise Inventory used in the British Cohort Study Age 46 survey

\section{Questions}

1. Do you feel tired most of the time?

2. Do you often feel miserable or depressed?

3. Do you often get worried about things?

4. Do you often get in a violent rage?

5. Do you often suddenly become scared for no good

6. Are you easily upset or irritated?

7. Are you constantly keyed up and jittery?

8. Does every little thing get on your nerves and wear you down?

9. Does your heart often race like mad?

Questions were asked in a yes/no format. A score of 0 was awarded for every 'no' response and a score of 1 awarded for every 'yes' response. 'Low malaise was defined as an overall score of 0-3, 'high malaise' was defined as an overall score $>3$. 
Supplementary Table 3: Mean differences in Warwick-Edinburgh Mental Wellbeing Scale with covariates

\begin{tabular}{|c|c|c|c|}
\hline & \multicolumn{3}{|c|}{ Mean difference in WEMWBS Score } \\
\hline & B & $95 \% \mathrm{Cl}$ & $\mathrm{p}$ \\
\hline Gender & 1.18 & $0.77-1.60$ & $<0.01$ \\
\hline Highest academic qualification & -0.02 & $-0.09-0.06$ & 0.71 \\
\hline Social occupational class & 0.01 & $-0.03-0.06$ & 0.54 \\
\hline Smoking & -0.57 & $-0.78--0.37$ & $<0.01$ \\
\hline BMI & -0.03 & $-0.07-0.01$ & 0.16 \\
\hline Disability & -2.10 & $-2.54--1.67$ & $<0.01$ \\
\hline Malaise score & -10.50 & $-11.08--9.93$ & $<0.01$ \\
\hline
\end{tabular}

WEMWBS, Warwick-Edinburgh Mental Wellbeing Scale. BMI, body mass index. Cl, confidence interval. 
Supplementary Table 4: Wellbeing in relation to common types of activity recorded via self-reported questionnaire

\begin{tabular}{|l|l|}
\hline TV VIEWING & Wellbeing score - B $(95 \% \mathrm{CI})^{*}$ \\
\hline$<1 \mathrm{hr} / \mathrm{d}$ & Ref \\
\hline $\mathbf{1 - 2} \mathrm{hr} / \mathrm{d}$ & $0.24(-0.22,0.69)$ \\
\hline$>2-4 \mathrm{hr} / \mathrm{d}$ & $-0.54(-1.0,-0.04)$ \\
\hline$\geq 4 \mathrm{hr} / \mathrm{d}$ & $-0.69(-1.21,-0.16)$ \\
\hline WALKING FOR PLEASURE & Wellbeing score B $(95 \% \mathrm{Cl}) *$ \\
\hline None & Ref \\
\hline 1 -2/month & $0.14(-0.29,0.56)$ \\
\hline $1-3 /$ week & $0.77(0.35,1.19)$ \\
\hline 4 or more per week & $0.60(0.15,1.04)$ \\
\hline
\end{tabular}

* B coefficient adjusted for sex, smoking, education, disability, self-rated health, malaise score, BMI 


\section{References}

\footnotetext{
${ }^{1}$ Keyes CLM, Annas J. Feeling good and functioning well: Distinctive concepts in ancient philosophy and contemporary science. The Journal of Positive Psychology. 2009;4:197-201.
}

${ }^{2}$ Ryan RM, Deci EL: On happiness and human potential: a review of research on hedonic and eudaimonic well-being. Annu Rev Psychol 2001, 52:141-166.

${ }^{3}$ World Health Organisation: Promoting Mental Health; Concepts emerging evidence and practice. Summary report Geneva; World Health Organisation; 2004.

${ }^{4}$ Chida Y, Steptoe A. Positive Psychological Well-Being and Mortality: A Quantitative Review of Prospective Observational Studies. Psychosomatic Medicine. 2008 Sep;70(7):741-756.

${ }^{5}$ Hamer, M., Endrighi, R. and Poole, L. (2012) 'Physical activity, stress reduction, and mood: insight into immunological mechanisms', Methods in Molecular Biology (Clifton, N.J.), 934, pp. 89-102. doi: 10.1007/978-1-62703-071-7_5.

${ }^{6}$ Pinto Pereira SM, Geoffroy M-C, Power C. Depressive symptoms and physical activity during 3 decades in adult life: bidirectional associations in a prospective cohort study. JAMA Psychiatry. 2014 Dec 1;71(12):1373-80.

${ }^{7}$ Stubbs B, Vancampfort D, Hallgren M, Firth J, Veronese N, Solmi M, et al. EPA guidance on physical activity as a treatment for severe mental illness: a meta-review of the evidence and Position Statement from the European Psychiatric Association (EPA), supported by the International Organization of Physical Therapists in Mental Health (IOPTMH). Eur Psychiatry. 2018;54:124-44.

${ }^{8}$ Buman MP, Hekler EB, Haskell WL, Pruitt L, Conway TL, Cain KL, et al. Objective Light-Intensity Physical Activity Associations With Rated Health in Older Adults. Am J Epidemiol. 2010 Nov 15;172(10):1155-65.

${ }^{9}$ Bell SL, Audrey S, Gunnell D, Cooper A, Campbell R. The relationship between physical activity, mental wellbeing and symptoms of mental health disorder in adolescents: a cohort study. Int J Behav Nutr Phys Act. 2019 Dec 26;16. 
${ }^{10}$ Withall J, Stathi A, Davis M, Coulson J, Thompson JL, Fox KR. Objective Indicators of Physical Activity and Sedentary Time and Associations with Subjective Well-Being in Adults Aged 70 and Over. Int J Environ Res Public Health. 2014 Jan;11(1):64356.

${ }^{11}$ Zhai, L., Zhang, Y. and Zhang, D. (2015) 'Sedentary behaviour and the risk of depression: a meta-analysis', British Journal of Sports Medicine. BMJ Publishing Group Ltd and British Association of Sport and Exercise Medicine, 49(11), pp. $705-709$. doi: 10.1136/bjsports-2014-093613.

12 Hamer M, Stamatakis E, Mishra GD. Television- and Screen-Based Activity and Mental Well-Being in Adults. American Journal of Preventive Medicine. 2010 Apr 1;38(4):375-80.

${ }^{13}$ Hamer M, Yates T, Sherar LB, Clemes SA, Shankar A. Association of after school sedentary behaviour in adolescence with mental wellbeing in adulthood. Preventive Medicine. 2016 Jun 1;87:6-10.

${ }^{14}$ Straatmann VS, Oliveira AJ, Rostila M, Lopes CS. Changes in physical activity and screen time related to psychological wellbeing in early adolescence: findings from longitudinal study ELANA. BMC Public Health. 2016 Sep 15;16.

${ }^{15}$ Okely JA, Čukić I, Shaw RJ, Chastin SF, Dall PM, Deary IJ, et al. Positive and negative well-being and objectively measured sedentary behaviour in older adults: evidence from three cohorts. BMC Geriatr. 2019 Jan 30;19.

${ }^{16}$ Chastin SFM, Dontje ML, Skelton DA, Čukid I, Shaw RJ, Gill JMR, Greig CA, Gale CR, Deary IJ, Der G, Dall PM; Seniors USP team. Systematic comparative validation of self-report measures of sedentary time against an objective measure of postural sitting (activPAL). Int J Behav Nutr Phys Act. 2018 Feb 26;15(1):21

${ }^{17}$ Ekelund U, Tarp J, Steene-Johannessen J, Hansen BH, Jefferis B, Fagerland MW, Whincup P, Diaz KM, Hooker SP, Chernofsky A, Larson MG, Spartano N, Vasan RS, Dohrn IM, Hagströmer M, Edwardson C, Yates T, Shiroma E, Anderssen SA, Lee IM. Dose-response associations between accelerometry measured physical activity and sedentary time and all cause mortality: systematic review and harmonised meta-analysis. BMJ. 2019 Aug 21;366:14570.

${ }^{18}$ Rosenberger ME, Haskell WL, Albinali F, et al. Estimating activity and sedentary behaviour from an accelerometer on the hip or wrist. Med Sci Sports Exerc. 2013;45(5):964-975. 
${ }^{19}$ Kozey-Keadle S, Libertine A, Lyden K, et al. Validation of wearable monitors for assessing sedentary behavior. Med Sci Sports Exerc. 2011;43(8):1561-1567.

20 16. Kozey-Keadle S, Libertine A, Staudenmayer J, Freedson P. The feasibility of reducing and measuring sedentary time among overweight, non-exercising office worker. J Obes, 2012;2012: 282-303.

${ }^{21}$ Elliott J, Shepherd P. Cohort profile: 1970 British Birth Cohort (BCS70). Int J Epidemiol. 2006;35(4):836-843.

22 Centre for Longitudinal Studies. 1970 British Cohort Study. https://cls.ucl.ac.uk/cls-studies/1970-british-cohortstudy/ Accessed 9 October 2019.

${ }^{23}$ Centre for Longitudinal Studies . BCS70 Age 46 Biomedical Sweep. https://cls.ucl.ac.uk/cls-studies/1970-british-cohortstudy/bcs70-age-46-biomedical-sweep/ Accessed 9 October 2019.

${ }^{24}$ Edwardson CL, Winkler EAH, Bodicoat DH, Yates T, Davies MJ, Dunstan DW, Healy GN. Considerations when using the activPAL monitor in field-based research with adult populations. J Sport Health Sci. 2017 Jun;6(2):162-178. doi: 10.1016/j.jshs.2016.02.002.

25 Hamer M, Stamatakis E, Chastin S, Pearson N, Brown M, Gilbert E, et al. Feasibility of measuring sedentary time with thigh worn accelerometry, and sociodemographic correlates: the 1970 British Cohort Study. [published online ahead of print, 2020 Mar 27]. Am J Epidemiol. 2020;kwaa047. doi:10.1093/aje/kwaa047

${ }^{26}$ Kim Y, Barry VW, Kang M. Validation of the ActiGraph GT3X and activPAL Accelerometers for the assessment of Sedentary Behavior. Meas Phys Ed Exerc Sci, 2015;19(3): 125-137.

${ }^{27}$ Tudor-Locke C, Aguiar EJ, Han H, Ducharme SW, Schuna JM Jr, Barreira TV, Moore CC, Busa MA, Lim J, Sirard JR, Chipkin SR, Staudenmayer J. Walking cadence (steps/min) and intensity in 21- 40 year olds: CADENCE-adults. Int J Behav Nutr Phys Act. 2019 Jan 17;16(1):8.

${ }^{28}$ Winkler EA, Bodicoat DH, Healy GN, Bakrania K, Yates T, Owen N, Dunstan DW, Edwardson CL. Identifying adults' valid waking wear time by automated estimation in activPAL data collected with a $24 \mathrm{~h}$ wear protocol. Physiol Meas. 2016 Oct;37(10):1653-1668. 
29 Tennant, R. et al. (2007) 'The Warwick-Edinburgh Mental Well-being Scale (WEMWBS): development and UK validation', Health and Quality of Life Outcomes, 5, p. 63. doi: 10.1186/1477-7525-5-63.

${ }^{30}$ Bass, M., Dawkin, M., Muncer, S., Vigurs, S. \& Bostock, J. Validation of Warwick Edinburgh Mental Well-being Scale (WEMWBS) in a population of people using Secondary Care Mental Health Services. Journal of Mental Health 25, 323-329 (2016).

${ }^{31}$ Bauman AE, Reis RS, Sallis JF, Wells JC, Loos RJ, Martin BW. Correlates of physical activity: why are some people physically active and others not? The Lancet. 2012 Jul 21;380(9838):258-71.

${ }^{32}$ Arora VS, Karanikolos M, Clair A, Reeves A, Stuckler D, McKee M. Data Resource Profile: The European Union Statistics on Income and Living Conditions (EU-SILC). Int J Epidemiol. 2015 Apr;44(2):451-61.

${ }^{33}$ Rutter M, Graham R Yule W (1970a) A neuropsychiatric study in childhood. London, Heinemann

${ }^{34}$ Rutter M, Tizard J, Whitmore K (1970 b) Education, health and behaviour. Longmans, London

35 McGee, R., Williams, S., Silva, P.A., 1986. An evaluation of the Malaise Inventory. J. Psychosom. Res. 30 (2), $147-152$.

${ }^{36}$ Bartram DJ, Yadegarfar G, Sinclair JMA, Baldwin DS. Validation of the Warwick-Edinburgh Mental Well-being Scale (WEMWBS) as an overall indicator of population mental health and well-being in the UK veterinary profession. The Veterinary Journal. 2011 Mar 1;187(3):397-8.

${ }^{37}$ Stamatakis E, Gale J, Bauman A, Ekelund U, Hamer M, Ding D. Sitting Time, Physical Activity, and Risk of Mortality in Adults. Journal of the American College of Cardiology. 2019 Apr 30;73(16):2062-72.

${ }^{38}$ Hallgren M, Dunstan DW, Owen N. Passive Versus Mentally Active Sedentary Behaviors and Depression. Exerc Sport Sci Rev. 2020 Jan;48(1):20-7.

${ }^{39}$ Atkin AJ, Adams E, Bull FC, Biddle SJ. Non-occupational sitting and mental well-being in employed adults. Ann Behav Med. 2012;43(2):181-188. doi:10.1007/s12160-011-9320-y 
40 Kandola A, Ashdown-Franks G, Hendrikse J, Sabiston CM, Stubbs B. Physical activity and depression: Towards understanding the antidepressant mechanisms of physical activity. Neurosci Biobehav Rev. 2019;107:525-539. doi:10.1016/j.neubiorev.2019.09.040

${ }^{41}$ Mandolesi L, Polverino A, Montuori S, et al. Effects of Physical Exercise on Cognitive Functioning and Wellbeing: Biological and Psychological Benefits. Front Psychol. 2018;9:509. Published 2018 Apr 27. doi:10.3389/fpsyg.2018.00509

42 Endrighi R, Steptoe A, Hamer M. The effect of experimentally induced sedentariness on mood and psychobiological responses to mental stress. Br J Psychiatry. 2016;208(3):245-251. doi:10.1192/bjp.bp.114.150755

${ }^{43}$ Hamer M, Sabia S, Batty GD, et al. Physical activity and inflammatory markers over 10 years: follow-up in men and women from the Whitehall II cohort study. Circulation. 2012;126(8):928-933. doi:10.1161/CIRCULATIONAHA.112.103879

44 Fancourt D, Steptoe A. The longitudinal relationship between changes in wellbeing and inflammatory markers: Are associations independent of depression?. Brain Behav Immun. 2020;83:146-152. doi:10.1016/j.bbi.2019.10.004

${ }^{45}$ Wosu AC, Valdimarsdóttir U, Shields AE, Williams DR, Williams MA. Correlates of cortisol in human hair: implications for epidemiologic studies on health effects of chronic stress. Ann Epidemiol. 2013;23(12):797-811.e2. doi:10.1016/j.annepidem.2013.09.006

${ }^{46}$ Firth J, Stubbs B, Vancampfort D, Schuch F, Lagopoulos J, Rosenbaum S, et al. Effect of aerobic exercise on hippocampal volume in humans: A systematic review and meta-analysis. Neuroimage. 2018 01;166:230-8.

${ }^{47}$ Kubarych TS, Prom-Wormley EC, Franz CE, Panizzon MS, Dale AM, Fischl B, et al. A multivariate twin study of hippocampal volume, self-esteem and well-being in middle-aged men. Genes, Brain and Behavior. 2012;11(5):539-44.

${ }^{48}$ Engeroff T, Füzéki E, Vogt L, Fleckenstein J, Schwarz S, Matura S, et al. Is Objectively Assessed Sedentary Behavior, Physical Activity and Cardiorespiratory Fitness Linked to Brain Plasticity Outcomes in Old Age? Neuroscience. 2018 15;388:384-92.

${ }^{49}$ Andersen MH, Ottesen L, Thing LF. The social and psychological health outcomes of team sport participation in adults: An integrative review of research. Scand J Public Health. 2019;47(8):832-850. doi:10.1177/1403494818791405 
${ }^{50}$ Isabelle Doré, Jennifer L. O'Loughlin, Mireille E. Schnitzer, Geetanjali D. Datta, Louise Fournier, The longitudinal association between the context of physical activity and mental health in early adulthood, Mental Health and Physical Activity. 2018;14:121-130.

${ }^{51}$ Larsen BA, Strong D, Linke SE. The association between family and friend integration and physical activity: results from the NHIS. Int J Behav Med. 2014 Jun;21(3):529-36.

52 McAneney H, Tully MA, Hunter RF, Kouvonen A, Veal P, Stevenson M, et al. Individual factors and perceived community characteristics in relation to mental health and mental well-being. BMC Public Health. 2015 Dec 12;15:1237.

53 Bryson A, Green F, Bridges S, Craig R. Health Survey of England: Wellbeing, health and work. 2010. http://www.hscic.gov.uk/catalogue/PUB03023/heal-surv-eng-2010-resp-heal-ch7-well.pdf. Accessed December 5, 2014.

${ }^{54}$ World Health Organization. Global Recommendations on Physical Activity for Health. Geneva: World Health Organization, 2010 http://whqlibdoc.who.int/publications/2010/9789241599979_eng.pdf?ua=1 . date last accessed: 1 August $2020 .$. 